\title{
La transparencia en la ley de contrataciones del Estado
}

\section{The transparency in state procurement law}

\author{
ANA TERESA REVILLA VERGARA*
}

Resumen: A partir de The Global Competitiveness Report 2010-2011, se presenta la situación del Perú a nivel internacional en cuatro indicadores relacionados al manejo de fondos públicos: desviación de fondos públicos; pagos irregulares y sobornos; favoritismo en las decisiones de los funcionarios del gobierno; y despilfarro del gasto público. Se compara al Perú con España y los países más grandes de América Latina, y se observa que está en un nivel intermedio, aunque por encima de la mayoría de los países de América Latina. También se identifican los principales aspectos que contribuyen a la transparencia en las compras públicas, ello a partir de la Ley de Contrataciones aprobada por el decreto legislativo 1017: la existencia del Registro Nacional de Proveedores; limitar la participación como proveedores del Estado hasta doce meses después de haber ejercido el cargo a los altos funcionarios del Estado, a sus parientes cercanos y a los altos funcionarios de los gobiernos regionales y locales en el gobierno regional o local en el que fueron autoridad; la obligatoriedad de que toda entidad pública cuente con un Plan Anual de Contrataciones, el Sistema Electrónico de Contrataciones del Estado y la prohibición del fraccionamiento. Se mencionan también otros aspectos de la normativa que no facilitarían la transparencia, entre los cuales está no registrar información por parte de las entidades públicas por los bienes y servicios adquiridos y obras ejecutadas por debajo de 3 unidades impositivas tributarias (UIT) —que en 2011 equivalen a S/. 10 800—, y se toma en cuenta, además, que estos son aproximadamente cien mil procesos.

Palabras clave: Corrupción - transparencia - fondos públicos - vigilancia social - Sistema Electrónico de Contrataciones del Estado - Plan Anual de Contrataciones - Registro Nacional de Proveedores - Tribunal de Contrataciones del Estado

Abstract: Since "The Global Competitiveness Report 2010-2011" the global situation of Peru is based on four indicators related to the management of public funds: deviation of public funds, irregular payments and bribes, favoritism in decisions of government officials, and wasteful public spending. Comparing Peru to Spain and the largest countries in Latin America, it was observed that Peru is at an intermediate level, but above most Latin American countries.

Based on the Procurement Law approved by the Legislative Decree 1017, the main aspects that contribute to transparency in public procurement can be

* Es abogada y egresada de la Maestría en Derecho Constitucional de la PUCP. Asimismo, se desempeña como docente en esa misma casa de estudios y es expositora acreditada, con gran experiencia en el sector público, del Organismo de Contrataciones del Estado (osce). Ha sido viceministra de Promoción del Empleo y de la Micro y Pequeña Empresa, asesora de la Presidencia de consucode, jefa del proyecto Transparencia en las Adquisiciones Estatales, entre otros. Actualmente integrante de la Oficina de Control de la Magistratura como representante de las facultades de derecho de las cinco universidades privadas más antiguas. 
identified: the existence of the National Registry of Suppliers, limiting participation as State providers until 12 months after having served State officials, their close relatives, and senior officials from regional and local governments in the Regional or Local Government in which they were authority; the requirement that every public entity has an Annual Procurement Plan, an State Procurement electronic system and fractionation prohibition. This article also mentions other legal aspects that could obstruct transparency such as not registering the information from public entities for goods and services purchased under 3 ITU (S/. 10.800), taking into account that these processes are approximately 100.000 .

Key words: Corruption - transparency - public funds - social monitoring state procurement electronic system - annual procurement plan- national register of suppliers - state procurement tribunal

\section{CONTENIDO: INTRODUCCIÓN.- I. EL PERÚ EN COMPARACIÓN CON OTROS PAÍSES DEL MUNDO.- II. LEY DE CONTRATACIONES DEL ESTADO.- III. CONCLUSIONES Y RECOMENDACIONES.}

\section{INTRODUCCIÓN}

La inexistencia de un buen sistema de vigilancia en el uso de los recursos públicos coadyuva a la creación de ambientes propicios para la corrupción. La Ley de Contrataciones del Estado ha introducido importantes mecanismos que permiten una adecuada supervisión, pero en otros casos los cambios producidos no favorecen la transparencia en las adquisiciones del Estado.

La construcción de un sistema de contrataciones en nuestro país ha permitido empezar a sentar las bases para facilitar el control social y reducir parcialmente la corrupción y orientación de los procesos de selección por parte de funcionarios del Estado encargados de las compras públicas. Este es un camino en el que hay mucho por hacer. Lo avanzado hasta el momento, sin embargo, permite observar y vigilar mejor los procesos de selección de las entidades públicas. Si bien puede darse el caso de autoridades que buscan la forma de "favorecer" con contratos del Estado a familiares, amigos, allegados o empresas que financiaron su campaña, esto puede ser, hoy en día, mejor fiscalizado y monitoreado por la ciudadanía, el periodismo de investigación y la Contraloría General de la República.

En el el cuarto informe cuatrimestral de marzo de $2011^{1}$, que aprobó la Comisión Especial Multipartidaria Permanente encargada del control, el seguimiento y la evaluación del Plan Nacional de Lucha contra la

1 En <http://www2.congreso.gob.pe/Sicr/ApoyComisiones/informes.nsf//nformesPorComisionEspecial /449CFC9835F6BF300525784F005D43B7/\$FILE/ATTLIHH3.pdf>. 
Corrupción del Congreso de la República, se señala que existen un total de 1365 procesos judiciales por presunta corrupción, reportados por la contraloría. Estos procesos involucran a 76581 funcionarios, de los cuales 218 son funcionarios del gobierno central; 13837 de los gobiernos regionales; y 62526 de los gobiernos locales. Estas cifras evidencian que hay un serio problema de corrupción especialmente con los funcionarios de los gobiernos regionales y locales. Si tomamos en cuenta el proceso de descentralización en el que se han transferido funciones y recursos a los gobiernos regionales y locales, vemos que el tema es especialmente relevante.

\section{EL PERÚ EN COMPARACIÓN CON OTROS PAÍSES DEL MUNDO}

El informe elaborado por el World Economic Forum, denominado The Global Competitiveness Report 2010-20112, nos permite evaluar la situación en la que se encuentra el Perú y compararla con otros (139) países del mundo. Consideramos de suma importancia esta herramienta, pues permite identificar el grado de avance o retroceso del Perú, así como compararlo con los demás. Este informe cuenta con doce indicadores básicos: destacan los de institucionalidad ${ }^{3}$, infraestructura, ambiente macroeconómico, salud y educación primaria e innovación. Dentro del indicador básico referido a la institucionalidad, encontramos los siguientes:

- desviación de fondos públicos ("1.03. Diversion of public funds");

- pagos irregulares o sobornos ("1.05 Irregular payments and bribes");

- favoritismo en las decisiones de los funcionarios del gobierno ("1.07 Favoritism in decisions of government officials"); y

- despilfarro del gasto público ("1.08 Wastefulness of government spending").

Considero relevante presentar la ubicación del Perú en cada uno de ellos, y compararlo a su vez con otros países de Latinoamérica y España.

\section{I.1. Desviación de fondos públicos ${ }^{4}$}

Frente a la pregunta de icuán común es la distracción de fondos públicos a empresas, individuos, o grupos debido a la corrupción en nuestro país?, considerando a 1 como "muy común", y 7 "como nunca sucede",

2 En <http://www3.weforum.org/docs/WEF_GlobalCompetitivenessReport_2010-11.pdf>.

3 El nombre del indicador es institutions.

4 Schwab, Klaus. "World Economic Forum. The Global Competitiveness Report 2010 -2011", p. 368. En <http://www3.weforum.org/docs/WEF_GlobalCompetitivenessReport_2010-11.pdf)>.

LATRANSPARENCIA

EN LA LEY DE

CONTRATACIONES

DEL ESTADO

THE

TRANSPARENCY

IN STATE

PROCUREMENT

LAW 
el país que mejor está considerado es Nueva Zelandia, con un puntaje 6,6. Perú aparece en el número 86 del ranking de un total de 139 países, con un puntaje de 3,1 .

Haciendo una comparación con otros países de América Latina, como se puede observar de la tabla que se presenta a continuación, vemos que Perú está por debajo de Uruguay Chile y Costa Rica, aunque por encima de México, Colombia, Brasil, Argentina, Ecuador, Venezuela y Paraguay.

\begin{tabular}{llc}
\hline Orden & \multicolumn{1}{c}{ País } & Puntaje \\
\hline 1 & Nueva Zelanda & 6,6 \\
30 & Uruguay & 5,1 \\
35 & Chile & 4,6 \\
49 & Costa Rica & 4,1 \\
50 & España & 4,0 \\
86 & Perú & 3,1 \\
98 & México & 2,8 \\
106 & Colombia & 2,6 \\
119 & Bolivia & 2,3 \\
121 & Brasil & 2,3 \\
123 & Argentina & 2,6 \\
135 & Ecuador & 2,0 \\
137 & Venezuela & 1,9 \\
138 & Paraguay & 1,9 \\
\hline
\end{tabular}

\section{I.2. Pagos irregulares y sobornos}

Este indicador representa el promedio de las siguientes preguntas de opinión:

¿En su país, cuán común es para las firmas hacer pagos adicionales o sobornos vinculados con?:

- importaciones y exportaciones;

- utilidades públicas;

- pago del impuesto anual;

- concesión de contraltos públicos y licencias;

- obtención de decisiones judiciales favorables. 
La respuesta a cada pregunta está entre los rangos de 1 (muy común) a 7 (nunca ocurre).

Aquí también observamos que el país mejor considerado es Nueva Zelandia, con un puntaje de 6.6. Perú se encuentra en el nivel medio: está en el puesto 66 de 139 países, con un puntaje de 4.1, por debajo de Chile, Uruguay, España y Costa Rica:

\begin{tabular}{llc}
\hline Orden & \multicolumn{1}{c}{ País } & Puntaje \\
\hline 1 & Nueva Zelanda & 6,6 \\
24 & Chile & 5,7 \\
32 & Uruguay & 5,4 \\
39 & España & 5,0 \\
52 & Costa Rica & 4,6 \\
66 & Perú & 4,1 \\
71 & Brasil & 4,0 \\
74 & El Salvador & 3,9 \\
90 & Colombia & 3,6 \\
91 & México & 3,6 \\
103 & Ecuador & 3,3 \\
115 & Argentina & 3,1 \\
120 & Bolivia & 2,9 \\
129 & Venezuela & 2,7 \\
131 & Paraguay & 2,7 \\
\hline
\end{tabular}

LATRANSPARENCIA

EN LA LEY DE

CONTRATACIONES

DEL ESTADO

THE

TRANSPARENCY

IN STATE

PROCUREMENT

LAW

\section{I.3. Favoritismo en las decisiones de los funcionarios del gobierno ${ }^{6}$}

Este indicador se encuentra más vinculado al tema de las compras gubernamentales. La pregunta que se formuló aquí estaba relacionada a icuán extendido está dentro de nuestro país las autoridades muestren favoritismo con firmas bien conectadas e individuos, cuando deciden sobre políticas y contratos públicos? La referencia es $1=$ siempre muestran favoritismo y 7 = nunca muestran favoritismo. 
Se puede observar que el país que mejor está es Suecia, con un puntaje de 6,0; Perú se encuentra en una ubicación intermedia, en el puesto 73 de 139 países, con un puntaje de 2,9, nuevamente por debajo de Chile, Uruguay, Costa Rica y España:

\begin{tabular}{llc}
\hline Orden & \multicolumn{1}{c}{ País } & Puntaje \\
\hline 1 & Suecia & 6,0 \\
21 & Chile & 44,3 \\
24 & Uruguay & 4,1 \\
39 & Costa Rica & 3,6 \\
57 & España & 3,2 \\
73 & Perú & 2,9 \\
74 & Brasil & 2,9 \\
83 & México & 2,8 \\
112 & Ecuador & 2,6 \\
118 & Colombia & 2,5 \\
128 & Bolivia & 2,3 \\
133 & Paraguay & 2,2 \\
135 & Venezuela & 2,0 \\
137 & Argentina & 1,9 \\
129 & Venezuela & 2,7 \\
131 & Paraguay & 2,7 \\
\hline
\end{tabular}

Este es un tema sobre el que nos queda todavía mucho por recorrer. A pesar de que hemos desarrollado un sistema de compras gubernamentales, se presentan situaciones en las que las autoridades del Estado no asumen un rol de independencia e imparcialidad, como debiera ser.

\subsection{Despilfarro del gasto público 7}

En relación con el empleo del gasto público, este indicador deja al Perú en una muy buena ubicación. Aquí figura como el segundo país latinoamericano, después de Chile. Si bien han existido importantes denuncias en contra de gobiernos locales por ejecutar algunas obras de poca o ninguna utilidad, no constituye la mayoría. 
Frente a la pregunta icómo considera usted el gasto público en su país?, tomando como 1 = sumamente derrochador y 7 = sumamente eficiente en el suministro de bienes necesarios y servicios, Perú está en el número 38 del ranking de 139 países, con un puntaje de 3,8. El país que mejor está es Singapur, con un puntaje de 6,1.

\begin{tabular}{llc}
\hline Orden & \multicolumn{1}{c}{ País } & Puntaje \\
\hline 1 & Singapur & 6,1 \\
26 & Chile & 4,2 \\
38 & Perú & 3,8 \\
46 & Costa Rica & 3,6 \\
81 & México & 3,1 \\
87 & Colombia & 3,0 \\
97 & Uruguay & 2,8 \\
101 & España & 2,7 \\
107 & Bolivia & 2,6 \\
127 & Paraguay & 2,2 \\
133 & Ecuador & 2,1 \\
134 & Argentina & 2,1 \\
136 & Brasil & 1,8 \\
137 & Venezuela & 1,7 \\
\hline
\end{tabular}

LATRANSPARENCIA

EN LA LEY DE

CONTRATACIONES

DEL ESTADO

THE

TRANSPARENCY

IN STATE

PROCUREMENT

LAW

Al parecer, los distintos sistemas de control de gasto impuestos en los últimos años en nuestro país por el Ministerio de Economía y Finanzas han dado un buen resultado. Un factor adicional que ha contribuido a esta situación puede ser la generación de un sistema de compras públicas en el país, que ha permitido disponer de mejores precios en bienes, servicios y obras adquiridas por entidades del sector público.

\section{LEY DE CONTRATACIONES DEL ESTADO}

El sistema de contrataciones que se ha venido impulsando en el país durante los últimos años intenta ordenar el tema de las adquisiciones para garantizar imparcialidad, transparencia y publicidad de los procesos de selección para adquirir bienes, servicios u obras ${ }^{8}$. Si bien en esto

8 El artículo 4 de la Ley de Contrataciones (decreto legislativo 1017) recoge los principios de promoción del desarrollo humano, moralidad, libre concurrencia y competencia, imparcialidad, razonabilidad, eficiencia, publicidad, transparencia, economía, vigencia tecnológica, equidad y sostenibilidad ambiental. 
no existe un nivel de consenso, pues hay quienes consideran que "estas medidas van en contra de la eficiencia y eficacia en la gestión administrativa pública al priorizar unos principios antes que otros y no tomar en cuenta aspectos importantes de la realidad del mercado peruano", es importante identificar qué normas de contrataciones favorecen efectivamente a la transparencia en los procesos de selección que realizan las Entidades del sector público y cuáles no.

\section{II.1. Aspectos que ayudan a la transparencia}

En el sistema de contrataciones peruano, hemos identificado algunos aspectos que consideramos de especial relevancia y que ayudan a la transparencia. Entre ellos están la existencia de un Registro Nacional de Proveedores; las limitaciones que se han señalado para ser contratista; la creación y la obligatoriedad del Plan Anual de Contrataciones del Estado; la creación del Sistema Electrónico de Contrataciones del Estado (seace); y la prohibición del fraccionamiento.

\section{II.1.1. Registro Nacional de Proveedores}

La existencia de un Registro Nacional de Proveedores en el cual deben inscribirse todas las personas naturales o jurídicas que desean participar como proveedores del Estado constituye una importante herramienta para la transparencia. Identificar quién o quiénes son proveedores de las entidades públicas permite hacer un mejor monitoreo sobre los proveedores, a fin de disponer de información de con quién se está contratando realmente — dado que no permite la suplantación de identidad— caso de incumplimiento por parte de los proveedores, posibilita aplicar las sanciones correspondientes.

Las entidades públicas carecían de información cierta del proveedor al que le adquirían bienes, servicios u obras. Esta situación se ha superado gracias a la existencia del registro. En el caso de que un proveedor presente información inexacta o documentación falsa, la ley (artículo 9) señala que se declara nula su inscripción en el registro. Los proveedores solo podrán solicitar su reinscripción en el referido registro luego de transcurridos dos años desde que quedó administrativamente firme la resolución que declaró la nulidad.

Una práctica común en el interior de las entidades públicas era tener un registro interno de los proveedores con los cuales trabajaban. Esto generaba muchos vínculos entre los proveedores y los responsables de las oficinas de logística y abastecimiento. Por ello, la Ley de Contrataciones ha dispuesto expresamente la prohibición de llevar un registro de proveedores a las entidades. De esta forma, se busca garantizar el

9 Tomamos como referencia las ideas del ingeniero Luis de la Flor, de la osce. 
principio de libre concurrencia y competencia consagrado en el artículo 4 de la Ley de Contrataciones del Estado. La idea de este principio es fomentar de manera más amplia, objetiva e imparcial la concurrencia, la pluralidad y la participación de postores.

La implementación del Registro Nacional de Proveedores constituye entonces un elemento de apoyo para las entidades públicas en la toma de decisiones de adquisiciones y contrataciones. Facilita la identificación y la validación de proveedores y simplifica la presentación de documentos por parte de ellos.

\section{II.1.2. Impedimento para ser contratista}

Otro elemento que suscita cierto nivel de discrepancia es el impedimento de participar como postores y/o contratista hasta doce meses después de haber dejado el cargo a autoridades y funcionarios de alto nivel. Esta norma se promulgó como respuesta al abuso que se produjo en nuestro país, por parte de quienes se aprovechaban de su situación privilegiada en un alto cargo público para beneficiarse directa o indirectamente ${ }^{10}$.

Los funcionarios de alto nivel que se encuentran bajo esta prohibición son "el Presidente y los Vicepresidentes de la República, los Congresistas, los Ministros y Viceministros, los Vocales de la Corte Suprema de Justicia, los titulares y los miembros del órgano colegiado de los Organismos Constitucionales Autónomos". Esta prohibición se extiende también "al cónyuge, conviviente o los parientes hasta el cuarto grado de consanguinidad y segundo de afinidad".

Esta norma se emitió con la idea de proteger también de situaciones de favoritismo a parientes cercanos entre los funcionarios de los gobiernos locales y regionales. La ley prohíbe que a nivel regional participen como postores o contratistas — hasta doce meses después de haber dejado el cargo - presidentes, vicepresidentes y consejeros de los gobiernos regionales; y, en el ámbito de su jurisdicción, los vocales de las cortes superiores de justicia, los alcaldes y regidores, así como sus cónyuges, convivientes o los parientes hasta el cuarto grado de consanguinidad y segundo de afinidad.

Están impedidos de ser proveedores del Estado, además las personas jurídicas y a las personas jurídicas sin fines de lucro:

g) En el ámbito y tiempo establecidos para las personas señaladas en los literales precedentes, las personas jurídicas en las que aquellas tengan o hayan tenido una participación superior al cinco por ciento (5\%) del 
capital o patrimonio social, dentro de los doce (12) meses anteriores a la convocatoria.

h) En el ámbito y tiempo establecidos para las personas señaladas en los literales precedentes, las personas jurídicas sin fines de lucro en las que aquellas participen o hayan participado como asociados o miembros de sus consejos directivos, dentro de los doce (12) meses anteriores a la convocatoria.

El objetivo de esta norma es limpiar el proceso de selección de todo interés que pueda interferir con los propios de la entidad para solicitar la adquisición de un bien o servicio, o la ejecución de una obra a una persona natural o jurídica independiente de todo vínculo familiar o de autoridad.

Sin embargo, a pesar de lo dispuesto en la Ley de Contrataciones y en su reglamento, al parecer se han producido situaciones que han evadido estos impedimentos. Así, en los considerandos del DS.046-2011-EF publicado recientemente ${ }^{11}$ "se ha observado que los proveedores que se encuentran impedidos para contratar con el estado vienen utilizando diversos mecanismos con el objeto de eludir dicha condición, por lo que es necesario establecer nuevos impedimentos", por lo cual se ha adicionado como impedimento, para ser participante, postor y/o contratista, a las personas naturales o jurídicas "a través de las cuales una autoridad, funcionario público, empleado de confianza, servidor público o proveedor pretenda eludir su condición de impedido valiéndose de cualquier modalidad de reorganización societaria y/o la utilización de testaferros para participar en un proceso de selección"12.

\section{II.1.3. Plan Anual de Contrataciones del Estado}

La obligatoriedad de contar con un Plan Anual de Contrataciones (pac), además de ser una excelente herramienta de planificación, es clave para la transparencia y permite hacer un control ciudadano. El pac debe ser aprobado por el titular de la entidad ${ }^{13}$. En él deben consignarse todos los bienes, servicios y obras que se requerirán durante el año fiscal, así como los montos estimados y los tipos de procesos de selección previstos, con independencia del régimen que las regule, o su fuente de financiamiento ${ }^{14}$. Este debe ser publicado en el Sistema Electrónico de Contrataciones del Estado (seace ${ }^{15}$.

11 Viernes 25 de marzo de 2011.

12 Artículo 2, en virtud del cual se modifica el artículo 237 del reglamento de la Ley de Contrataciones del Estado.

$13 \mathrm{Al}$ ser el titular de la entidad el responsable de la aprobación del pac, se garantiza que este tiene conocimiento de todos los bienes, servicios y obras que se van a convocar durante el ejercicio presupuestal, por lo que se lo responsabiliza de las decisiones referidas a estos campos.

14 Artículo 8 de la Ley de Contrataciones del Estado (decreto legislativo 1017).

15 En <http://www.seace.gob.pe>. 
La introducción del pac causó mucho revuelo en el interior de las entidades del sector público, a pesar de que se realizó en forma gradual. En un primer momento, la elaboración del plan anual de contrataciones fue voluntaria y se presentaba de manera física. Es aquí cuando el Consejo Superior de Contrataciones y Adquisiciones del Estado (consucode), hoy Organismo Supervisor de las Contrataciones del Estado (osce), realizó un proceso de difusión masiva sobre la necesidad de su elaboración, acompañado de un permanente proceso de capacitación y monitoreo de entidades para asesorarlas en su elaboración. En un segundo momento, y ya con la implementación del seace, se dispuso su obligatoriedad.

Para impulsar su obligatoriedad, se estableció la nulidad de oficio, la convocatoria y/o realización de cualquier licitación pública, concurso público, adjudicación directa pública o selectiva y adjudicación de menor cuantía (programable) que haya sido efectuada sin la aprobación de pac correspondiente al año fiscal en curso o que no haya sido programada e incluida en dicho pac (artículo 3, directiva 005-2009-osce/CD).

Hoy en día, prácticamente todas las entidades públicas (más de 2600 ) cumplen con la obligación de publicar el Plan Anual de Contrataciones en el Sistema Electrónico de Contrataciones del Estado, lo que evidentemente constituye un avance importante en la planificación que realizan las entidades y en la transparencia de la gestión.

Desde la creación del pac, el número de planes registrados en consucode - en un primer momento- y posteriormente inscritos en el seace ha venido en aumento año a año:

\begin{tabular}{cc}
\hline Año & $\begin{array}{c}\text { Número de planes anuales } \\
\text { inscritos en osce }\end{array}$ \\
\hline 2002 & 387 \\
2003 & 805 \\
2004 & 987 \\
2005 & 1592 \\
2006 & 1941 \\
2007 & 2348 \\
2008 & 2534 \\
2009 & 2585 \\
2010 & 2603 \\
2011 & $2492^{*}$ \\
\hline
\end{tabular}

Fuente: Elaboración propia sobre datos del seace.

LATRANSPARENCIA

EN LA LEY DE

CONTRATACIONES

DEL ESTADO

THE

TRANSPARENCY

IN STATE

PROCUREMENT

LAW 
Como podemos observar, al inicio fueron muy pocas las entidades que publicaron sus planes anuales de contrataciones, pero una vez que esto fue obligatorio, y gracias a la difusión realizada, podemos afirmar que prácticamente el universo de los funcionarios de las oficinas de administración o logística de las entidades saben que deben elaborar el pac e incluir en él los procesos de selección.

La publicación en el pac ${ }^{16}$ permite que los ciudadanos podamos observar qué bienes, obras o servicios que están programando adquirir o contratar los ministerios, los gobiernos locales y los gobiernos regionales, y de qué tipo de proceso se trata. Si es una menor cuantía, una adjudicación directa selectiva o pública, un concurso público o una licitación pública, cuánto está programando gastar tentativamente la entidad en la adquisición de dicho bien o servicio o en la construcción de la obra, para cuándo está programando la entidad su convocatoria y con qué fuente de financiamiento cuenta ${ }^{17}$.

El 2010 se registraron más de 110 mil procesos de selección en los planes anuales de contrataciones de las entidades del sector público:

\section{Número de procesos de selección registrados en los planes anuales de} contrataciones de las entidades del sector público

\begin{tabular}{|c|c|c|c|c|c|c|c|c|c|c|}
\hline 㝴 & 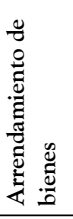 & 氖 & $\begin{array}{l}0 \\
\tilde{\pi} \\
0 \\
0 \\
0 \\
0 \\
0 \\
0 \\
5 \\
0 \\
0 \\
0\end{array}$ & 胥 & 莣 & 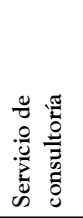 & 总 & 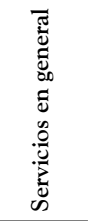 & 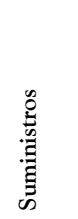 & $\frac{\mathscr{g}}{\tilde{\pi}}$ \\
\hline 2004 & 334 & 18719 & 850 & 6030 & 306 & 2130 & 11229 & 1365 & 3182 & 44145 \\
\hline 2005 & 1284 & 46979 & 1887 & 10281 & 534 & 7144 & 4807 & 31945 & 408 & 105269 \\
\hline 2006 & 1526 & 59714 & 2923 & 11757 & 560 & 9884 & 5922 & 43535 & 0 & 135821 \\
\hline 2007 & 1537 & 74041 & 5152 & 16181 & 564 & 11266 & 98 & 45869 & 0 & 154708 \\
\hline 2008 & 1711 & 94378 & 7972 & 18131 & 563 & 15337 & 2486 & 58254 & 0 & 198834 \\
\hline 2009 & 0 & 67605 & 8851 & 17735 & 0 & 0 & 39959 & 0 & 0 & 134150 \\
\hline 2010 & 0 & 57009 & 5743 & 14258 & 0 & 0 & 33264 & 0 & 0 & 110274 \\
\hline 2011 & 0 & 29914 & 2267 & 9138 & 0 & 0 & 21748 & 0 & 0 & 63067 \\
\hline
\end{tabular}

Fuente: Elaboración propia sobre datos del seace al 10 de mayo de 2011.

16 En <http://www.seace.gob.pe/Default.asp?_pageid_=20\&_contentid_=79>.

17 Como mínimo, el Plan Anual de Contrataciones debe contar con el siguiente contenido: el objeto de la contratación, la descripción de los bienes, servicios u obras a contratar y el correspondiente código asignado en el catálogo, el valor estimado de la contratación, el tipo de proceso que corresponde al objeto y su valor estimado - así como la modalidad de selección, la fuente de financiamiento, el tipo de moneda, los niveles de centralización o desconcentración de la facultad de contratar, y la fecha prevista de la convocatoria (artículo 7 del reglamento de la Ley de Contrataciones, decreto supremo 184-2008-EF). 
La obligatoriedad de registrar el plan anual por parte de las entidades públicas fue lo que produjo un salto en el número de procesos registrados, entre un año (2004) y otro (2005).

También se puede observar el crecimiento sustancial del número total de procesos de selección realizado por las aproximadamente 2700 entidades del estado. Dicho aumento alcanza su pico en 2008 y decae en 2009. Esto se debe a que, en febrero de aquel año, entró en vigencia el decreto legislativo 1017, que dispone que se encuentran al margen de la Ley de Contrataciones del Estado los procesos menores a 3 UIT y el decreto legislativo 1057 en virtud del cual se regula el régimen especial de contratación administrativa de servicios y se modifican los contratos de servicios no personales que tenían suscritas muchas personas que trabajaban en la administración pública ${ }^{18}$.

El valor estimado de los procesos de selección que figuran en los planes anuales de contrataciones de las entidades del sector público estuvo el año pasado en cerca de 46 mil millones de soles (ver tabla de página siguiente).

Debido a la publicación de los procesos de selección en el pac, por ejemplo, sabemos que para este año 2011:

- El Congreso de la República ha registrado que va a utilizar S/. 26702802 para la adquisición de bienes, servicios y obras. Tiene programada una licitación pública por la suma de S/. 1275755 para la adquisición de cartuchos de tinta, tóner para impresora y CD ROM. Asimismo, para el mes de agosto, se ha programado la adquisición de 130 medallas para los nuevos congresistas, a un valor estimado de S/. 140010 (lo que equivale a más de mil soles por medalla); una licitación pública para la adquisición de papel bond por S/. 729 806; un software de interconectividad de plataformas (solución de integración de sistemas) por $\mathrm{S} / .855000$, entre otros ${ }^{19}$.

- La sede central del gobierno regional de Ancash, de un monto total de su plan anual de S/. $22421562144^{20}$, tiene programada

18 La cuarta disposición complementaria final señala que "Las entidades comprendidas en la presente norma quedan prohibidas en lo sucesivo de suscribir o prorrogar contratos de servicios no personales o de cualquier modalidad contractual para la prestación de servicios no autónomos. Las partes están facultadas para sustituirlos antes de su vencimiento, por contratos celebrados con arreglo a la presente norma".

19 En <http://www.seace.gob.pe/Default.asp?scriptdo=PKU_PROCESOS_PLAN_ANUAL.doview\&ag_ eue_codigo $=6 \&$ ag_anho $=20118 \mathrm{ag} \_$codigo $=1 \& \_C A L I F I C A D O R \_=P O R T L E T .1 .103 .0 .20 .79 \&$ REGIONID_=1\&_PORTLETID_=103\&_ORDERID_=0\&_PAGEID_=20\&_CONTENTID_=79\&_ USERID_=<!--USERID-->\&_PRIVILEGEID_=1>.

$20 \mathrm{En} \quad<$ http://www.seace.gob.pe/Default.asp?_CALIFICADOR_=PORTLET.1.103.0.20.79\& REGIONID_=1\&_PORTLETID_=103\&_PRIVILEGEID_=1\&_ORDERID_=0\&_PAGEID_=20\&_ CONTENTID_=79\&_USERID_=\%3C\%21--USERID--\%3E\&_EVENTNAME_=\&_OBJECTFIRE_=\&_ OBJECTEVENT_=\&scriptdo=PKU_PLAN_ANUAL.doview\&ag_anho=2011\&ag_desc $=$ gobierno+regi onal+de+ancash>.

LATRANSPARENCIA

EN LA LEY DE

CONTRATACIONES

DEL ESTADO

THE

TRANSPARENCY

IN STATE

PROCUREMENT

LAW 


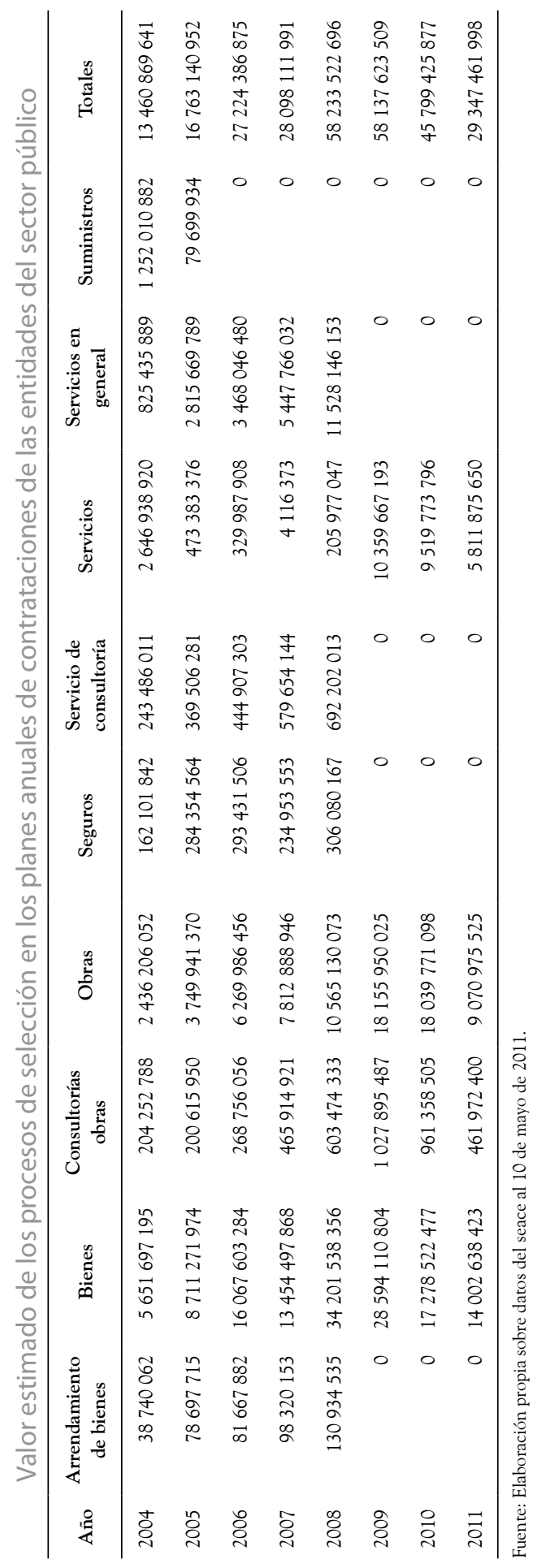


la ejecución de 199 procesos de elección entre los que encontramos la ejecución de importantes obras de infraestructura ${ }^{21}$.

- Echarate, del valle de la Convención, departamento del Cus$\mathrm{co}$, al ser rico en yacimientos de hidrocarburos, es el distrito con mayor canon minero. De un presupuesto ascendente a S/. 19162 698,08, se puede observar que las autoridades del gobierno local, en lugar de realizar procesos de selección para la ejecución de las obras, han decidido hacerlas ellos mismos. Así, entre los procesos de selección, podemos observar que para el mes de febrero tenía programada una subasta inversa electrónica para adquirir petróleo D-2 y gasolina de 84 y de 90 octanos por un monto ascendente a S/. 2017613 "para varias obras"; la realización de un concurso público por un monto ascendente a S/. 450000 para el servicio de alquiler de volquete 15 metros cúbicos que serviría durante la obra de mejoramiento y construcción de la carretera Miraflores-Manto Real; la contratación del servicio de un tractor oruga de 220 caballos de fuerza por un monto estimado de S/. 192 000; la contratación del servicio de cargador frontal de 160-195 caballos de fuerza por un monto de S/. 100 000; la adquisición de cemento Portland para la obra de mejoramiento y construcción la carretera Miraflores-Manto Real por un monto estimado de S/. 154 000, entre otros.

De esta información se deduce que la municipalidad está optando por realizar ella directamente las obras, lo cual implica alquilar los servicios diversos y los insumos que para ello se requieran. Ello, en nuestra opinión, no es lo más apropiado, por la desnaturalización de funciones que se produce y porque, en términos generales, las obras cuestan y tardan más. Muchos gobiernos locales, sin embargo, siguen esta modalidad de "administración directa" para ejecutar obras con la intención de dar trabajo a la población de la localidad.

\section{II.1.4. Sistema Electrónico de Contrataciones del Estado}

Este sistema ha sido fundamental para permitir el acceso a todos los ciudadanos de la vigilancia sobre los procesos de contratación pública en

21 Entre ellas, pueden citarse el mejoramiento de la capacidad resolutiva de los servicios del puesto de salud (Jimbe) del distrito de Cáceres del Perú (Santa, Ancash), por un valor estimado de S/. 2501 595,25; la construcción y el mejoramiento de la infraestructura educativa del Instituto Superior Tecnológico Eleazar Guzmán Barrón (Huaraz, Ancash), por un monto estimado de S/. 4337 211,85; la reconstrucción y el equipamiento de la institución educativa pública Santa Inés (distrito de Yungay, Ancash), por un monto estimado de S/. 11203 387,80; el mejoramiento y la ampliación del sistema de riego Ruricocha (distrito de Acochaca, provincia de Asunción en la región Ancash), por un monto estimado de S/. 5135 355,58; el mejoramiento del sistema de irrigación Mishacocha-Cashan-Allma (provincia de Huaraz, Ancash), por un monto estimado de S/. 4726 293,62. En <http://www.seace. gob.pe/Default.asp?scriptdo=PKU_PROCESOS_PLAN_ANUAL.doview\&ag_eue_codigo $=33 \&$ ag anho $=2011 \&$ ag_codigo $=1 \&$ ag_desc_proc $=\% \& a v \_p r o c \_n a m e=\& a g \_c u r r e n p a g e=1 \&$ _CALIFIC

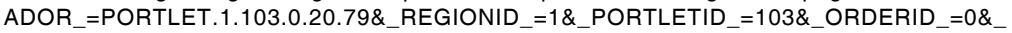
PAGEID_=20\&_CONTENTID_=79\&_USERID_=<!--USERID-->\&_PRIVILEGEID_=1 . 
nuestro país. El seace es "el sistema electrónico que permite el intercambio de información y difusión sobre las contrataciones del Estado, así como la realización de transacciones electrónicas"22. Es desarrollado, administrado y operado por el Organismo Supervisor de la Contrataciones del Estado ${ }^{23}$.

Las entidades que se encuentran comprendidas ${ }^{24}$ dentro de los alcances de la Ley de Contrataciones están obligadas a registrar en el seace todos los actos realizados en cada proceso de selección que convoque, los contratos suscritos y su ejecución, bajo responsabilidad. Las entidades que están exceptuadas de registrar información en el seace están obligadas a remitir a la Contraloría General de la República una relación de todas las convocatorias realizadas en dicho período, con la documentación que permita apreciar su resultado, dentro de los quince días siguientes al cierre de cada trimestre ${ }^{25}$ :

La obligatoriedad de publicidad en el seace incluye a las contrataciones que realicen las entidades cualquiera fuese el régimen legal o fuente de financiamiento al que se sujete la contratación estatal. La información que se registra en el seace debe ser idéntica a aquella que se tiene como documento final para la realización de cualquier acto ya sea en el proceso de selección contrato o su ejecución; bajo responsabilidad del funcionario que hubiese solicitado la activación del Certificado seace y de aquél que hubiera registrado la información ${ }^{26}$.

En este sistema electrónico se consignan, además de los Planes Anuales de Contrataciones, las bases, la absolución de consultas, la absolución de observaciones a ellas, los pronunciamientos que hubiesen, las bases integradas, el cuadro comparativo y/o las actas en virtud de las cuales se otorga la buena pro al postor ganador, la resolución de los recursos de apelación, y la resolución de recursos de revisión — si los hubiese-.

Antes de la existencia del seace, los únicos que accedían a las bases de un proceso de selección eran "los postores"; ello limitaba enormemente la participación de posibles postores que no se enteraron del proceso. Hoy en día, al ser un sistema de acceso público, permite fiscalizar e identificar posibles actos de corrupción o inconducta funcional. Han sido

22 Artículo 67, decreto legislativo 1017.

23 Artículo 68 del decreto legislativo 1017.

24 Estas son (a) el gobierno nacional, sus dependencias y reparticiones; (b) los gobiernos regionales, sus dependencias y reparticiones; (c) los gobiernos locales, sus dependencias y reparticiones; (d) los organismos constitucionales autónomos; (e) las universidades públicas; (f) las sociedades de beneficencia y las juntas de participación social; (g) las fuerzas armadas y la Policía Nacional del Perú; (h) los fondos de salud, de vivienda, de bienestar y demás de naturaleza análoga de las fuerzas armadas y de la Policía Nacional del Perú; (i) las empresas del Estado de Derecho público o privado, ya sean de propiedad del gobierno nacional, regional o local, y las empresas mixtas bajo control societario del Estado; y (j) los proyectos, programas, fondos, órganos desconcentrados, organismos públicos del Poder Ejecutivo, instituciones y demás unidades orgánicas, funcionales, ejecutoras y/o operativas de los poderes del Estado, así como los organismos a los que alude la Constitución Política del Perú y los demás que sean creados y reconocidos por el ordenamiento jurídico nacional. 25 Artículo 45. Ley de contrataciones del Estado (decreto legislativo 1017).

26 R. 424-2010-OSCE-PRE, numeral VI-E (aprueban directiva 008-2010/OSCE-CD, que establece disposiciones aplicables al registro de información de los procesos de selección y otros actos en el Sistema Electrónico de Contrataciones del Estado [seace]). 
muchos los casos en los que los periodistas de investigación han identificado "procesos dirigidos" o a un postor beneficiado, de un momento a otro, con muchos procesos.

La ley obliga a las entidades públicas a utilizar el seace "sin perjuicio de la utilización de otros regímenes especiales de contratación estatal, según se establezca en el Reglamento" (artículo 68).

Señala además que "El Reglamento establecerá los criterios de incorporación gradual de las Entidades al Sistema Electrónico de Contrataciones del Estado (seace), considerando la infraestructura y condiciones tecnológicas que éstas posean o los medios disponibles para estos efectos" (artículo 68). En el seace aparece el registro de la cantidad de procesos convocados desde 2005 a la fecha.

En lo que se refiere a los montos de los procesos convocados, podemos observar que ha ido en permanente aumento: su pico se registra en 2009, año en el que gobierno dictó una serie de medidas para impulsar el gasto público a fin de que esto redunde en la economía del país.

El año 2010 registra menos convocatorias informadas también por el aumento a 3 UIT de los procesos que están fuera de la Ley de Contrataciones.

Comparando la información registrada por las entidades del sector público sobre el valor estimado de los procesos de selección en los planes anuales de contrataciones con los montos de las convocatorias informadas, tenemos lo siguiente:

\begin{tabular}{lcc}
\hline & $\begin{array}{c}\text { Valor estimado de los procesos de } \\
\text { selección en los planes anuales de } \\
\text { contrataciones de las entidades del } \\
\text { sector público }\end{array}$ & $\begin{array}{c}\text { Convocatorias informadas } \\
\text { por tipo de entidad / } \\
\text { Montos convocados }\end{array}$ \\
\hline 2004 & 13460869641 & $* *$ \\
2005 & 16763140952 & 12145825723 \\
2006 & 27224386875 & 18648862946 \\
2007 & 28098111991 & 18939506498 \\
2008 & 58233522696 & 41851876628 \\
2009 & 58137623509 & 55139057823 \\
2010 & 45799425877 & 40661540458 \\
$2011^{* * *}$ & 29347461998 & 13172012463 \\
\hline
\end{tabular}

* Estas cifras corresponden a los procesos convocados, no a los que fueron adjudicados.

** No se registran datos.

*** al 10 de mayo de 2011.

LATRANSPARENCIA

EN LALEY DE

CONTRATACIONES

DEL ESTADO

THE

TRANSPARENCY

IN STATE

PROCUREMENT

LAW 


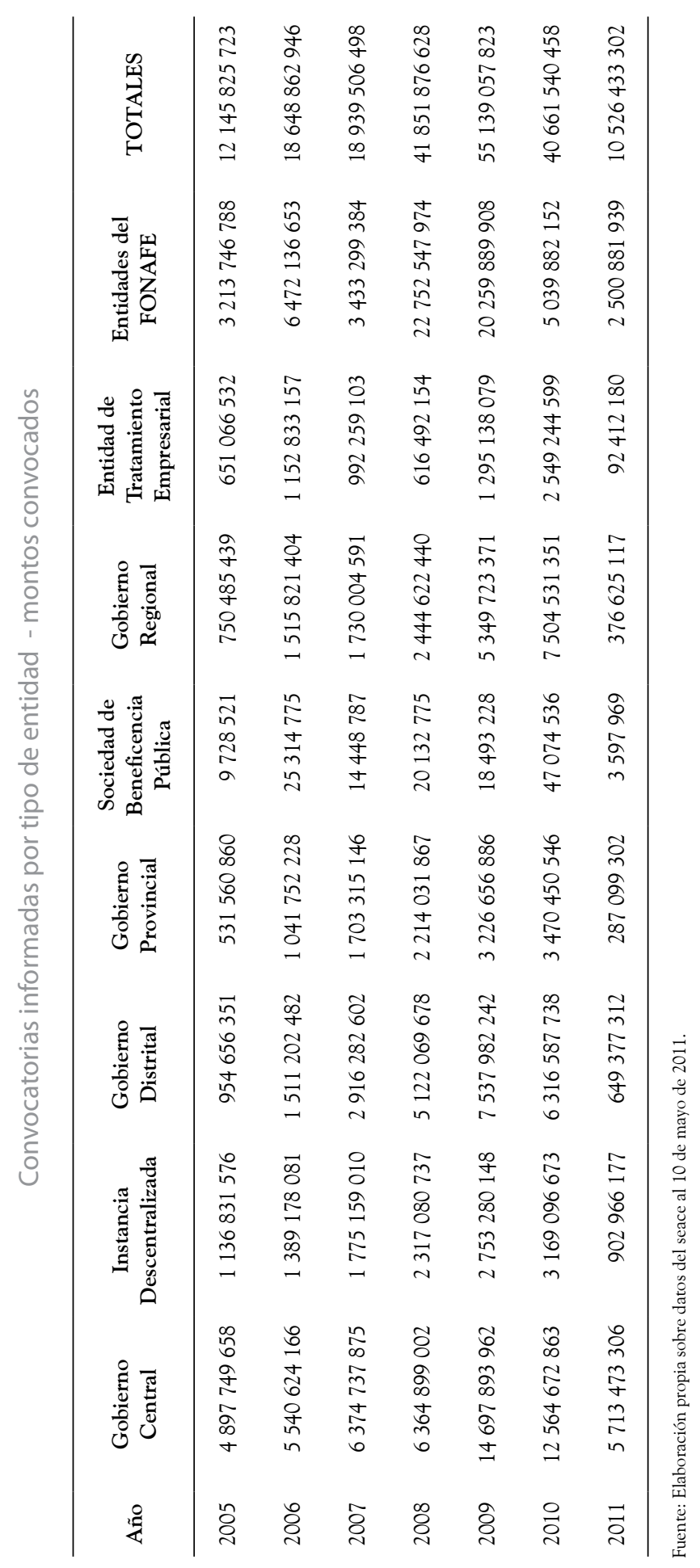


Se observa una importante diferencia entre lo señalado en el plan anual y lo efectivamente convocado. Las cifras entre lo programado y realmente convocado se acercan mucho más en 2009. Las diferencias que se observan en 2011 obedecen a que los procesos todavía no han sido convocados.

El seace es una herramienta de muchísima utilidad. Considero que ha sido uno de los más grandes aciertos para uniformizar procesos, transparentar los procesos de contrataciones, promover la participación de pluralidad de postores y permitir la vigilancia social.

\section{II.1.5. La prohibición del fraccionamiento}

Es clave para evitar la distorsión de los tipos procesos de selección. Las entidades deben planificar la adquisición de bienes, servicios y obras por lo menos para un año ${ }^{27}$. Esta planificación permite reducir el número de procesos de contratación, pero sobre todo permite reducir costos por volumen, lo que favorece a la economía de la propia entidad.

La ley de Contrataciones del Estado prohíbe expresamente el fraccionamiento:

Artículo 19.- Prohibición de fraccionamiento

Queda prohibido fraccionar la contratación de bienes, de servicios y la ejecución de obras con el objeto de modificar el tipo de proceso de selección que corresponda, según la necesidad anual. No se considera fraccionamiento a las contrataciones por etapas, tramos, paquetes o lotes posibles en función a la naturaleza del objeto de la contratación o para propiciar la participación de las pequeñas y micro empresas en aquellos sectores económicos donde exista oferta competitiva.

Por "fraccionar" se entiende la adquisición de los mismos bienes en más de una oportunidad durante un mismo ejercicio fiscal. En esta figura también se puede caer con la adquisición de servicios u obras que se "dividen" para modificar el tipo de proceso de selección al que le correspondería el íntegro del bien, servicio u obra que requiere la entidad.

Los tipos del proceso de selección son menor cuantía, adjudicación directa selectiva, adjudicación directa pública, concurso público, y licitación pública. Es importante identificar el valor referencial del bien, servicio u obra, a fin de estimar correctamente el tipo de proceso de selección a seguir.

La ley de presupuesto establece anualmente los valores para cada proceso. Para el año fiscal 2011, ha fijado los siguientes valores:

LATRANSPARENCIA

EN LA LEY DE

CONTRATACIONES

DEL ESTADO

THE

TRANSPARENCY

IN STATE

PROCUREMENT

LAW 


\begin{tabular}{|c|c|c|c|c|}
\hline Tipo de proceso & & Bienes & Servicios & Obras \\
\hline Licitación pública & & $>=400000$ & & $>=1800000$ \\
\hline Concurso público & & & $=400000$ & \\
\hline \multirow[t]{4}{*}{ Adjudicación directa } & Pública & $<$ de 400000 & $<\operatorname{de} 400000$ & < de 1800000 \\
\hline & & > de 200000 & $>$ de 200000 & > de 900000 \\
\hline & Selectiva & $<=$ de 200000 & $<=$ de 200000 & $<=$ de 900000 \\
\hline & & $>=$ de 40000 & $>=$ de 40000 & $>=$ de 180000 \\
\hline \multirow[t]{2}{*}{ Menor cuantía } & & $<\operatorname{de} 40000$ & $<$ de 40000 & < de 180000 \\
\hline & & $>$ de 10800 & $>$ de 10800 & > de 10800 \\
\hline
\end{tabular}

Con la prohibición del fraccionamiento de los procesos de selección, se busca que los bienes, servicios u obras que requiere una entidad sean adquiridos conforme lo disponen las normas de contrataciones para todos los procesos de esa misma envergadura. El órgano encargado de las contrataciones en cada entidad está obligado de garantizar el cumplimiento de estas disposiciones.

\section{II.2. Aspectos que no ayudan a la transparencia \\ II.2.1. Está fuera de los alcances de la Ley de Contrataciones la adquisición de bienes servicios u obras que estén por debajo de 3 Unidades Impositivas Tributarias}

La Ley de Contrataciones del Estado aprobada mediante el decreto legislativo 1017 trajo como una de sus novedades el aumentar de 1 a 3 UIT la adquisición de bienes, servicios y obras que se encuentran al margen de la Ley de Contrataciones del Estado. Hoy en día ello equivale a S/. $10800^{28}$.

Esto fue muy bien recibido por los funcionarios públicos, pues les otorga un mayor nivel de discrecionalidad en la toma de decisiones al flexibilizar la norma, lo cual les permite a las entidades ejecutar de una manera rápida, ágil y directa:

[...] hasta antes de la vigencia de la LCE, los contratos administrativos que oscilaban entre montos mayores a una (1) UIT y menores a tres (3) UITs [sic]. Significaban un porcentaje importante dentro del conjunto de las contrataciones que realiza el Estado, de tal manera, que ahora en adelante, existirá un gran número de convocatorias que no serán publi-

28 "Artículo3 inc.3.3 [...] la presente norma no es de aplicación para: [...] h) Las contrataciones cuyos montos, sean iguales o inferiores a tres (3) Unidades Impositivas Tributarias, vigentes al momento de la transacción; salvo que se trate de bienes y servicios incluidos en el Catálogo de Convenios Marco". 
cadas en el seace, originando con ello que diversos postores no puedan acceder a dichas convocatorias, lo cual al no generarse competencia, las entidades no obtendrán los mejores precios del mercado sobre un bien, simplemente porque dichos precios no resultan competitivos ${ }^{29}$.

Va a depender de los funcionarios encargados de las adquisiciones el promover la diversidad de proveedores. Es muy importante que se difunda el resultado de estas decisiones, pues, al no hacerlo, no se transparenta la gestión ni permite la vigilancia ciudadana.

Como podemos observar, las adjudicaciones de menor cuantía (AMC) se han reducido de 234021 en 2008 a 78250 (33,4\% menos) en 2009 y a 57023 (24\% menos) en 2010. Son más de 170000 procesos que escapan a la ley de contrataciones, dentro de los cuales están los contratos Contratos Administrativos de Servicios, que se estiman en 70 000. Esto no se condice con el principio de libre concurrencia y competencia a que hace referencia el artículo 4, inciso c, de la ley, que señala: "En los procesos de contrataciones se incluirán regulaciones o tratamientos que fomenten la más amplia, objetiva, e imparcial concurrencia, pluralidad y participación de postores".

Al haber eliminado de la aplicación de la ley a esos procesos y al no publicarse la decisión de los funcionarios, se establecen vínculos con un grupo selecto de proveedores y ello limita la participación de otros. Si bien puede ser una medida que facilita la gestión, sería conveniente evaluar los mecanismos de publicidad de estas operaciones para impulsar una mayor participación de proveedores e impulsar la transparencia.

\section{II.2.2. Las entidades - $-\mathrm{y}$ no el osce - se pronuncian respecto de los cuestionamientos formulados a las bases que no fueron acogidos por el Comité Especial, siempre que el valor referencial del proceso sea inferior a 300 UIT}

Las bases constituyen la parte más importante del proceso de selección. Estas son aprobadas por el titular de la entidad o por el funcionario a quien le hayan delegado dicha facultad. Ellas deben contener los mecanismos que fomenten la mayor concurrencia y participación de postores en función a:

- el objeto del proceso y la obtención de la propuesta técnica y económica más favorable;

- el detalle de las características técnicas de los bienes, servicios u obras a contratar;

LATRANSPARENCIA

EN LA LEY DE

CONTRATACIONES

DEL ESTADO

THE

TRANSPARENCY

IN STATE

PROCUREMENT

LAW 


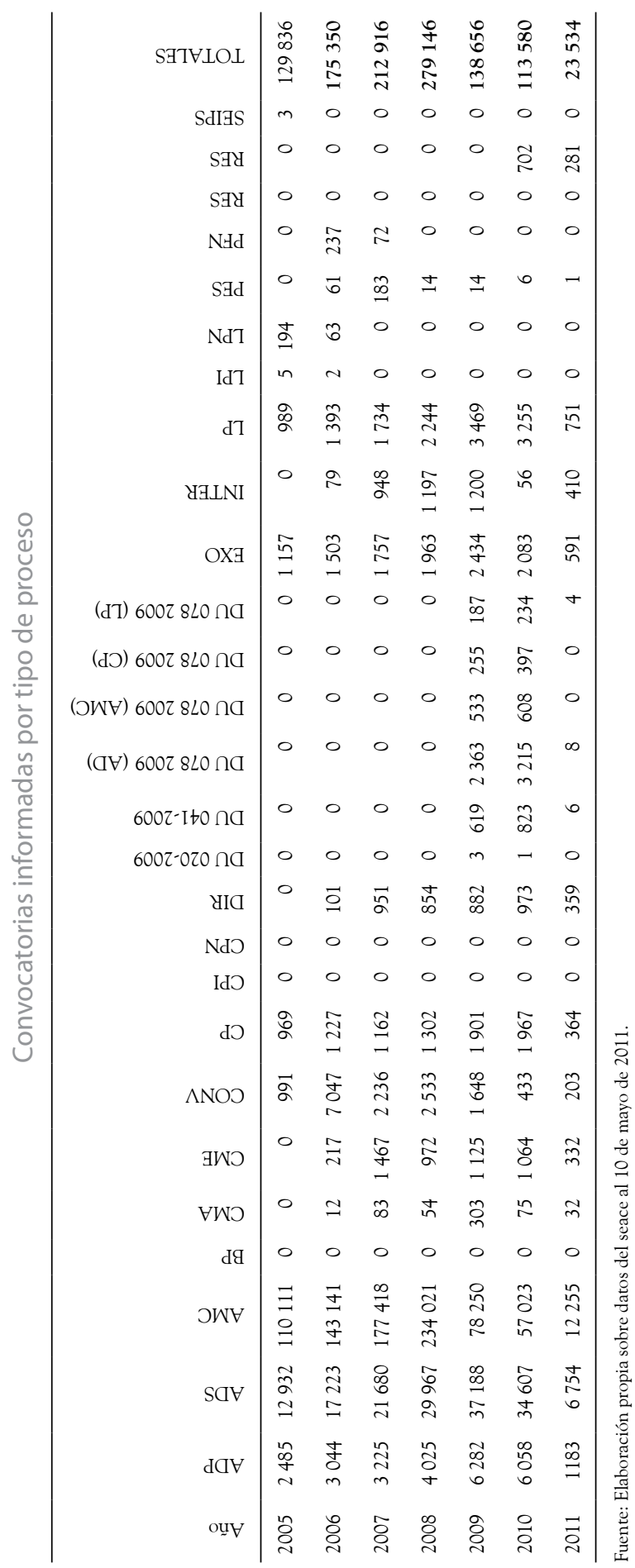


- el lugar de entrega, elaboración o construcción, así como el plazo de ejecución, según el caso;

- las garantías;

- los plazos y mecanismos de publicidad, la definición del sistema y/o modalidad a seguir;

- el método de evaluación y calificación de propuestas;

- la proforma de contrato, el valor referencial, los mecanismos que aseguren la confidencialidad de las propuestas; y

- el cronograma del proceso de selección ${ }^{30}$.

El cronograma de las bases debe establecer un plazo para la presentación de consultas y observaciones a su contenido, así como otro plazo para su absolución.

Mediante las consultas, se formulan pedidos de aclaración a las disposiciones de las bases, y a través de las observaciones los postores pueden cuestionarlas en lo relativo al incumplimiento de las condiciones mínimas o de cualquier disposición en materia de contrataciones del Estado u otras normas complementarias o conexas que tengan relación con el proceso de selección.

La absolución de las consultas y observaciones deben ser absueltas por el Comité Especial mediante un pliego por escrito, debidamente fundamentado y sustentado, sea que las acoja, lo haga parcialmente o no lo haga. El pliego deberá contener la identificación de cada participante que las formuló, las consultas presentadas y la respuesta para cada una de ellas. Deberá ser notificado a través del seace y a los correos electrónicos de los participantes, de ser el caso (reglamento, artículo 54). Tanto las respuestas a las consultas como las observaciones a las bases que se acogieron se consideran como parte integrante de ellas ${ }^{31}$.

Si alguno de los postores no está de acuerdo con la respuesta del Comité Especial, la anterior Ley de Contrataciones señalaba que se podía apelar esta decisión al consucode ${ }^{32}$; no lo veía la misma entidad, sino que se derivaba a un organismo externo.

30 Artículo 26 del decreto legislativo 1017.

31 Artículo 28 del decreto legislativo 1017.

$32 \mathrm{El}$ anterior reglamento de la Ley de Contrataciones del Estado (decreto supremo 084-2004PCM), como su modificatoria (decreto supremo 107-2007-EF), señalaban que: "Artículo $116^{\circ}$.Elevación de observaciones al consucode. Los observantes tienen la opción de solicitar quelas Bases y los actuados del proceso sean elevados al consucode dentro de los tres (3) días hábiles siguientes al vencimiento del término para absolverlas. Dicha opción no solo se originara cuando las observaciones formuladas no sean acogidas por el Comité Especial, sino, además, cuando el mismo observante considere que el acogimiento declarado por el Comité Especial continua siendo contrario a lo dispuesto por el artículo $25^{\circ}$ de la Ley, cualquier otra disposición de la normativa sobre contrataciones y adquisiciones del Estado $\mathrm{u}$ otras normas complementarias o conexas que tengan relación con el proceso de selección" (subrayado nuestro).

LATRANSPARENCIA

EN LA LEY DE

CONTRATACIONES

DEL ESTADO

THE

TRANSPARENCY

IN STATE

PROCUREMENT

LAW 
La ley vigente ha otorgado esta facultad al titular de la entidad en última instancia, siempre que el valor referencial del proceso de selección sea igual o menor a 300 UIT $^{33}$ :

Artículo 28.- [...]

En caso que el Comité Especial no acogiera las observaciones formuladas por los participantes, éstos podrán solicitar que las Bases y los actuados del proceso sean elevados al Organismo Supervisor de las Contrataciones del Estado - osce, siempre que el Valor Referencial del proceso de selección sea igual o mayor a trescientas (300) Unidades Impositivas Tributarias (UIT).

Si el Valor Referencial es menor al monto señalado en el párrafo precedente, las observaciones serán absueltas por el Titular de la Entidad en última instancia.

El procedimiento y plazo para tramitar las consultas y observaciones se fijará en el Reglamento.

$\mathrm{Al}$ restarle al osce la facultad de pronunciarse en todos los casos que los postores observan las bases, se limita su actuación para aquellos en los que el valor referencial es igual o mayor a 300 UIT. Ello ha generado un giro de 180 grados con relación al rol que venía desempeñando como unificador de criterios, con una consecuente dispersión de respuestas por las entidades. Como señala Martínez Zamora:

[...] no es difícil adivinar que progresivamente veremos una proliferación de requisitos particulares, exigencias desproporcionadas o excluyentes y, en general, una acelerada diferenciación adversa de las bases que aprueben las entidades, que serán las únicas encargadas de resolver las controversias en la formulación de las bases en todos los procesos que alcancen hasta las 300 UIT. Resulta dudoso, en este sentido, que el control disuasivo que ejercía la sola posibilidad de elevar las bases al consucode pueda ser suplida con controles posteriores o denuncias, sin una presencia definida como etapa del proceso de selección. Se rompe con ello el sueño de criterios de interpretación uniformes y requisitos homogéneos, aspecto que no podrá ser cumplido por el método de control ex post que ahora caracteriza el régimen de contratación pública ${ }^{34}$.

Dado que cada entidad va a resolver según su propio criterio, se pueden llegar a generar respuestas contradictorias ante los mismos problemas. Los proveedores tendrán una multiplicidad de criterios de interpretación, pues solo llegarán al osce las observaciones que se planteen en un número reducido de procesos de selección. Esta norma 
ha reducido sustancialmente la actuación del osce respecto de los cuestionamientos formulados a las bases que no fueron acogidos por el Comité Especial.

\section{II.2.3. Las entidades resuelven en única y última instancia administrativa los recursos que se interpongan, derivados de los procesos de selección siempre que el valor referencial de los mismos no supere las 600 UIT}

Con la nueva ley de contrataciones, se ha reducido también sustancialmente la competencia del Tribunal de Contrataciones del Estado para resolver las discrepancias que surjan entre la entidad y los participantes o postores en un proceso de selección. Mediante el recurso de apelación, se pueden impugnar los actos dictados desde la convocatoria de un proceso de selección hasta antes de la celebración del contrato. El recurso de apelación sólo podrá interponerse luego de otorgada la buena pro.

Esta norma genera dos situaciones. Primero, le otorga a la misma la entidad la facultad de resolver la mayoría de los casos en los que se produzcan impugnaciones. Con esta situación pueden registrarse entidades que no actúen con la imparcialidad requerida. De otro lado, se produce una gran diversidad de respuestas, algunas de ellas contradictorias, por parte de las más de 2700 entidades en los casos que se interpongan recursos de apelación. Esta nueva ley (artículo 53) señala que "el recurso de apelación será conocido y resuelto por el Titular de la Entidad siempre y cuando el valor referencial del proceso no supere las seiscientas (600) Unidades Impositivas Tributarias (UIT). En caso el valor referencial del proceso de selección sea superior a dicho monto, los recursos de apelación serán conocidos y resueltos por el Tribunal de Contrataciones del Estado".

Entonces, solo los casos en los que el proceso de selección tenga un valor referencial igual o mayor a 600 UIT —es decir, el equivalente a 2160000 soles durante 2011 — son vistos por el Tribunal de Contrataciones del Estado ${ }^{35}$.

Los actos impugnables dentro de un proceso de selección son:

- los dictados por el Comité Especial o el órgano encargado de las contrataciones, según corresponda, durante el desarrollo del proceso de selección;

- los expedidos luego de haberse otorgado la buena pro y hasta antes de la celebración del contrato; 
- los emitidos por el titular de la entidad que afecten la continuación del proceso de selección, distintos de aquellos que resuelven recursos de apelación, tales como nulidad de oficio, cancelación $\mathrm{u}$ otros $^{36}$.

Este recurso solo puede interponerse luego de otorgada la buena pro.

Con la nueva ley se aumentó también la garantía que se exige para la interposición del recurso de apelación. Se subió de 1\% a 3\% del valor referencial del proceso de selección o del ítem que se decida impugnar. En cualquier caso, la norma afirma que la garantía no podrá ser menor al 50\% de 1 UIT. La garantía debe otorgarse en favor del osce y de la entidad, cuando corresponda.

En opinión de Córdova Schaefer: "La intención de este dispositivo fue la de reducir los incentivos de interposición de recursos impugnativos maliciosos o temerarios, pero también desalienta al postor que compite legítimamente, mientras que la Entidad tiene el incentivo de negar dichos recursos en vista que se queda con el depósito de la garantía"37.

La decisión de esta norma de limitar la actuación del Tribunal de Contrataciones del Estado, así como la de trasladar esta competencia a las propias entidades que son las que llevan los procesos de selección, no ayuda a la transparencia. No se va a producir una unidad de criterio para resolver controversias similares y, al ser la misma entidad la que resuelve, corre el riesgo de perder imparcialidad frente a lo resuelto por el Comité Especial.

\section{CONCLUSIONES Y RECOMENDACIONES}

1. Al encontrarse en una situación intermedia en comparación con los otros países del mundo en relación con los indicadores de "desviación de fondos públicos", "pagos irregulares y sobornos", "despilfarro del gasto público" y "favoritismo en las decisiones de los funcionarios del gobierno", el Perú está mejor ubicado que muchos de los países latinoamericanos. Se encuentra, sin embargo, por debajo de Chile, Uruguay, España y Costa Rica, lo que evidencia que en este aspecto hay todavía mucho por hacer.

2. Gracias a la obligatoriedad de la inscripción de los planes anuales de contratación, al Sistema Electrónico de Contrataciones del Estado, al Registro Nacional de Proveedores y a la existencia de normas que referidas a la prohibición de ser contratistas a los parientes de altos funcionarios y la prohibición del fraccionamiento, el Estado peruano 
cuenta con importantes herramientas que permiten a cualquier ciudadano monitorear y fiscalizar el uso de los recursos públicos.

3. No obstante, algunas modificaciones recientes, orientadas a otorgar mayor discrecionalidad a los funcionarios sobre los recursos públicos, afectan la transparencia. Aldejar al margen de la Ley de Contrataciones aquellas adquisiciones que estén por debajo de 3 UIT (S/. 10 800), se han reducido en más de 100 mil los procesos registrados en el seace. Queda pendiente una modificación normativa que al menos obligue la inscripción en el seace del producto adquirido, el monto pagado por la entidad, la oportunidad en que se realizó el pago y el proveedor beneficiado.

4. Es importante evaluar la decisión de trasladar a las entidades y no al osce las observaciones a las bases y los recursos de impugnación por nuevos requisitos que algunas entidades puedan establecer de manera arbitraria, así como la eliminación de criterios de interpretación uniformes. 